\title{
Synchrotron radiation X-ray diffraction of modulated structures in charge-density-wave materials. Application to $\mathrm{NbSe}_{3}$
}

\author{
S. VAN SMAALEN, J.L. DE BOER and P. COPPENS* \\ Laboratory of Chemical Physics, Materials Science Center, University of Groningen, Nijenborgh 4, \\ 9747 AG Groningen, The Netherlands \\ " Department of Chemistry, State University of New York at Buffalo, Buffalo, NY 14214, U.S.A.
}

\begin{abstract}
Synchrotron radiation X-ray diffraction has been performed on niobium triselenide at $20 \mathrm{~K}$. The modulation parameters belonging to both Charge-Density-Waves (CDW's) have been determined. The high-temperature $\mathrm{CDW}$ is found to comprise of displacements on all atoms of column III, as well as on Se atoms of column II. Similarly, the low-temperature CDW resides on column I and also involves displacements of some $\mathrm{Se}(\mathrm{II})$. The structure is interpreted in terms of CDW's on $\mathrm{Nb}$ (III) and $\mathrm{Nb}(\mathrm{I})$, and elastic coupling between the atoms. The correlations between the atomic displacements are studied by analyzing the correlations between the varying interatomic distances. The atomic valences are discussed in the framework of the Bond-Valence method.
\end{abstract}

\section{Introduction}

On lowering the temperature, transition metal trichalcogenides, $\mathrm{MX}_{3}$, and related ternary compounds, often transform into an incommensurate Charge-Density-Wave (CDW) state [1]. Primarily, the interest in these compounds is focused on peculiar properties of the CDW state, e.g. the non-linear electrical conductivity. Prime example is $\mathrm{NbSe}_{3}$, which goes through two independent $\mathrm{CDW}$ transitions, at $\mathrm{T}_{\mathrm{c} 1} \simeq 145 \mathrm{~K}$ and $\mathrm{T}_{\mathrm{c} 2}=59 \mathrm{~K}$, respectively, and shows prominent nonlinear conductivity [2]. Understanding the properties of the $\mathrm{CDW}$ state requires detailed knowledge of the accompanying structural distortions, as discussed in this paper.

The trichalcogenides usually grow as thin needle-shaped crystals. Furthermore, the atomic displacements related to the CDW are small, so that the scattering from the modulation wave is weak, and conventional $\mathrm{X}$-ray sources are insufficient for measurement of the intensities. Synchrotron radiation provides the necessary increased intensity and improved signal to noise ratio. It also allowes tuning of the wavelength such that absorption can be minimized, thus facilitating the interpretation of the scattered intensities from these highly anisotropically shaped crystals.

The basic structure of $\mathrm{NbSe}_{3}$ is known at $100 \mathrm{~K}$ and at room temperature [3]. It can be characterized as a collection of columns of face sharing triangular prisms of selenium atoms. Each prism accommodates a Niobium atom at its center. Neighboring columns are shifted over one half lattice parameter, resulting in a eight-fold coordination of $\mathrm{Nb}$ by Se. There are three crystallographically independent columns in the unit cell, labelled I, II and III, respectively 


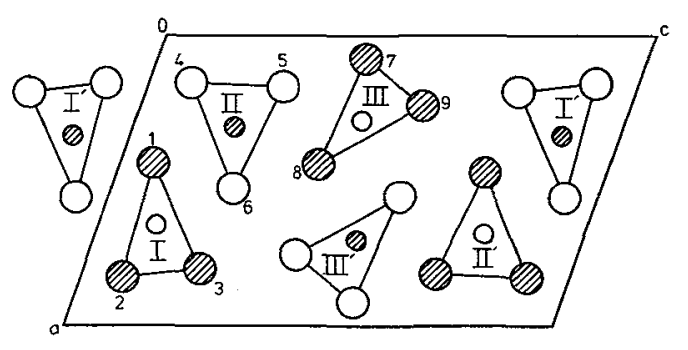

Figure 1. Projection of the structure of $\mathrm{NbSe}_{3}$ along the monoclinic unique b-axis. Large circles correspond to Se, small circles denote $\mathrm{Nb}$ atoms. Open circles have $\mathrm{y}=0.25$, hatched circles have $y=0.75$. Roman numbers represent column types as well as the $\mathrm{Nb}$ atoms.

(Fig. 1).

The lattice distortions connected with the CDW's have been observed by the presence of satellite reflections in electron diffraction [4] and $X$-ray diffraction [5]. The highertemperature CDW corresponds to a modulation wave vector $\vec{q}_{1}=(0,0.241,0)$; the lowertemperature CDW corresponds to $\overrightarrow{\mathrm{q}}_{2}=(0.5,0.260,0.5)$. Precise values for the incommensurate components were obtained in high resolution $X$-ray scattering, using synchrotron radiation [6]. It was found that $2\left(q_{1} y+q_{2 y}\right)$ significantly differs from one, thus showing the independent character of both CDW's.

Synchrotron radiation X-ray diffraction has been performed on $\mathrm{NbSe}_{3}$ and $\mathrm{NiTa}_{2} \mathrm{Se}_{7}$ at $20 \mathrm{~K}$. The modulation parameters have been determined belonging to both CDW's in $\mathrm{NbSe}_{3}$, using the superspace symmetry [7]. The structure was interpreted in terms of CDW's on $\mathrm{Nb}(\mathrm{III})$ and $\mathrm{Nb}(\mathrm{I})$, and displacements of $\mathrm{Se}$ due to elastic coupling between the atoms. In the present paper the modulation is analyzed in terms of varying interatomic distances, and the effect of the modulation on the atomic valences is discussed, using the Bond-Valence method.

\section{Experimental}

Two needle shaped crystals were selected for the synchrotron experiment [8]. Crystal 1 was of dimensions $15 \mu \mathrm{m} \times 25 \mu \mathrm{m}$ along a $\times$ c. Its length along the monoclinic b-axis was $3 \mathrm{~mm}$. The corresponding dimensions for crystal 2 were $20 \mu \mathrm{m} \times 30 \mu \mathrm{m}$ and $3 \mathrm{~mm}$ long. For both crystals the cross section perpendicular to $\vec{B}$ had the shape of a parallelogram, with an angle of 110 degree, equal to the angle of the unit cell.

X-ray diffraction experiments were done on beam line X3A of the National Synchrotron Light Source at Brookhaven National Laboratory, New York, U.S.A.. Monochromatized radiation was obtained with a silicon monochromator, using the Si(111) reflection. A wave length of $\lambda=1.0001(1) \AA$ was selected, in order to minimize absorption. The width was $\Delta \lambda=0.0014 \AA$. The crystals were mounted on a helium cryostat, with the long axis parallel to the $\phi$ angle. The cryostat was kept at a temperature of $20 \mathrm{~K}$. The intensity of the incident radiation was continuously monitored by an ionization chamber positioned between the monochromator and the crystal. These values were used to correct the measured counts. Furthermore, corrections were 
applied for the dead time of the counter, and for the Lorentz and polarization effects. Absorption correction was applied $\left(\mu=119.9 \mathrm{~cm}^{-1}\right)$, with minimum/maximum correction factors of $0.71 / 0.85$ for crystal 1 and $0.69 / 0.81$ for crystal 2. Standard deviations in the intensities were calculated based on counting statistics.

Lattice parameters were determined from 38 main reflections, measured on crystal 1 . They are essentially in agreement with Hodeau et al. [3]. At $20 \mathrm{~K}$ they were obtained as: $a=9.974(1) \AA$, b $=3.476(2) \AA, c=15.592(2) \AA$, and $\beta=109.41(1)$ degree. Satellite reflections were observed at the positions $\vec{q}_{1}=(0,0.241,0)$ and $\vec{q}_{2}=(0.5,0.260,0.5)$. No attempt was made to determine new accurate values for the incommensurate parameters.

The scattered intensities of main reflections and first-order satellites were measured up to $\theta=28$ degree. The total width of each scan was 2 degree, with a time spend of $60 \mathrm{~s}$ for main reflections, $120 \mathrm{~s}$ for $\mathbf{q}_{1}$ type satellites and $240 \mathrm{~s}$ for the $\mathbf{q}_{2}$ type satellites. For crystal 1 the data collected comprise the $k=1, k=2$, and $k=3$ layers. The $k=0$ layer was measured on crystal 2. The intensity control reflections showed variations in time up to a factor of three. The reflections in each time-slice were scaled according to the three intensity control reflections in their group. Subsequently, equivalent reflections were averaged in Laue symmetry $2 / \mathrm{m}$. This reduced 1957 reflections measured on crystal 1 to 1856 unique ones, with an internal consistancy of $2 \%$. For crystal 2, 217 reflections were merged into 159 unique ones, with an internal consistancy of $4 \%$. The resulting data sets were used for structure determination and refinements (details are given in Table 1).

\section{Structure determination}

All reflections can be indexed by five integers with respect to the reciprocal axes of the basic structure and both modulation wavevectors. To facilitate the analysis, a larger basic structure unit cell was chosen as $\mathbf{A}=\mathbf{a}+\mathbf{c}, \mathbf{B}=\mathbf{b}$, and $\mathbf{C}=-\mathbf{a}+\mathbf{c}$. This transforms the second modulation wave vector to one without the commensurate components:

$$
\mathbf{q}_{2}^{\prime}=(0,0.261,0)
$$

With respect to $\mathbf{q}_{1}$ and $\mathbf{q}_{2}^{\prime}$ and the transformed reciprocal basis, the indexing becomes:

$$
S=\mathrm{H}_{1} \mathbf{A}^{*}+\mathrm{H}_{2} \mathbf{B}^{*}+\mathrm{H}_{3} \mathrm{C}^{*}+\mathrm{H}_{4} \mathbf{q}_{1}+\mathrm{H}_{5} \mathbf{q}_{2}
$$

The transformation introduces a general extinction condition, given by $\mathrm{H}_{1}+\mathrm{H}_{3}+\mathrm{H}_{5}=$ odd is absent for the $\left(\mathrm{H}_{1}, \cdots, \mathrm{H}_{5}\right)$ reflections.

From the diffraction symmetry, and the extinction conditions, the superspace symmetry was determined as $P 2_{1} / \mathrm{m}(0 \quad \beta 0)\left(1 / 2 \beta^{\prime} 1 / 2\right)$ [7]. With respect to the larger unit cell, this $(3+2)$ dimensional superspace group is defined by the elements $(E 11)=\left(x_{1}, x_{2}, x_{3}, x_{4}, x_{5}\right),\left(2_{1} s 1\right)$ $=\left(-x_{1}, 0.5+x_{2},-x_{3}, 0.5+x_{4}, x_{5}\right)$, (i I 1$)=\left(-x_{1},-x_{2},-x_{3},-x_{4},-x_{5}\right),(m$ I 1$)=\left(x_{1}, 0.5-x_{2}\right.$, $\left.x_{3}, 0.5-x_{4},-x_{5}\right)$, and the centering translation ct $=(0.5,0,0.5,0.5,0)$. Note that $s$ 
Table 1. R-factors for the final refinement. Partial $R$-factors for various subsets of reflections are also given, they are defined as $R_{F}=\left(\Sigma|| F_{0}|-| F_{c}||\right) /\left(\Sigma\left|F_{\mathrm{o}}\right|\right)$ and $w R=$ $\left(\left(\Sigma w\left|F_{o}-F_{c}\right|^{2}\right) /\left(\Sigma w\left|F_{o}\right|^{2}\right)\right)^{1 / 2} . F_{o}$ and $F_{c}$ are the observed and calculated structure factors, respectively; $w$ is the weight.

\begin{tabular}{cccccccc}
\hline & \multicolumn{3}{c}{ crystal 1 } & \multicolumn{4}{c}{ crystal 2 } \\
reflection set & $\begin{array}{c}\text { number of } \\
\text { reflections }\end{array}$ & RF & wR & number of \\
& 917 & 0.077 & 0.114 & 99 & 0.055 & 0.091 \\
\hline observed & 917 RF & wR \\
main reflections & 378 & 0.073 & 0.111 & 86 & 0.055 & 0.091 \\
q1 satellites & 433 & 0.115 & 0.123 & 10 & 0.117 & 0.101 \\
q2 satellites & 162 & 0.180 & 0.164 & 3 & 0.335 & 0.335 \\
\hline
\end{tabular}

Table 2. Basic structure coordinates and temperature factors as obtained from the refinement of the complete structure. Position parameters are relative to the larger unit cell. Temperature factors have to be multiplied by $10^{-5}$, and are given in $\AA^{2}$. Note that $\mathrm{U}_{12}$ and $\mathrm{U}_{23}$ are zero by symmetry. Standard deviations in the last digits are given in parentheses.

\begin{tabular}{lccccccc}
\hline Atom & $\mathrm{x}_{1}^{\mathrm{o}}$ & $\mathrm{x}_{2}^{\mathrm{o}}$ & \multicolumn{1}{c}{$\mathrm{x}_{3}^{\mathrm{o}}$} & \multicolumn{1}{c}{$\mathrm{U}_{11}$} & \multicolumn{1}{c}{$\mathrm{U}_{22}$} & \multicolumn{1}{c}{$\mathrm{U}_{33}$} & $\mathrm{U}_{13}$ \\
\hline $\mathrm{Nb} 1$ & $0.3858(1)$ & 0.25 & $-0.2674(1)$ & $32(11)$ & $87(14)$ & $-34(41)$ & $-37(15)$ \\
$\mathrm{Nb2}$ & $0.2615(1)$ & 0.75 & $-0.0552(1)$ & $52(11)$ & $57(14)$ & $-07(42)$ & $-58(15)$ \\
$\mathrm{Nb3}$ & $0.3780(1)$ & 0.25 & $0.0837(1)$ & $31(10)$ & $86(14)$ & $-63(40)$ & $-15(14)$ \\
Se1 & $0.2539(2)$ & 0.75 & $-0.1868(1)$ & $40(12)$ & $61(17)$ & $123(57)$ & $-53(19)$ \\
Se2 & $0.4601(2)$ & 0.75 & $-0.3733(1)$ & $61(14)$ & $45(16)$ & $33(53)$ & $-74(19)$ \\
Se3 & $0.5212(2)$ & 0.75 & $-0.2826(1)$ & $74(14)$ & $93(16)$ & $-01(56)$ & $-48(21)$ \\
Se4 & $0.1260(2)$ & 0.25 & $-0.0392(1)$ & $74(15)$ & $136(17)$ & $-29(59)$ & $-62(21)$ \\
Se5 & $0.2273(2)$ & 0.25 & $0.0491(1)$ & $75(14)$ & $75(16)$ & $60(53)$ & $-95(20)$ \\
Se6 & $0.3881(2)$ & 0.25 & $-0.1397(1)$ & $57(14)$ & $70(17)$ & $47(55)$ & $-75(21)$ \\
Se7 & $0.2534(2)$ & 0.75 & $0.1716(1)$ & $50(13)$ & $111(17)$ & $-39(56)$ & $-87(21)$ \\
Se8 & $0.4246(2)$ & 0.75 & $-0.0195(1)$ & $84(13)$ & $102(16)$ & $-86(51)$ & $-52(18)$ \\
Se9 & $0.4099(2)$ & 0.75 & $0.1658(1)$ & $68(15)$ & $134(17)$ & $-104(55)$ & $-50(21)$ \\
\hline
\end{tabular}

Table 3. Modulation parameters obtained from the refinement of the complete structure. Values are defined according to Eq.3, but are given in Ångstrom along the supercell axes. Standard deviations in the last digit are in parentheses. The left three columns give the $\mathbf{q}_{1}$-type modulation, the three right most columns give the $\mathbf{q}_{2}$ modulation.

\begin{tabular}{lcccccr}
\hline Atom & \multicolumn{1}{c}{$\mathrm{B}_{10}^{\mathrm{x}}$} & \multicolumn{1}{c}{$\mathrm{A}_{10}^{\mathrm{y}}$} & \multicolumn{1}{c}{$\mathrm{B}_{10}^{\mathrm{z}}$} & \multicolumn{1}{c}{$\mathrm{A}_{01}^{\mathrm{x}}$} & \multicolumn{1}{c}{$\mathrm{B}_{01}^{\mathrm{y}}$} & \multicolumn{1}{c}{$\mathrm{A}_{01}^{\mathrm{z}}$} \\
\hline $\mathrm{Nb} 1$ & $0.002(1)$ & $0.003(1)$ & $-0.004(1)$ & $0.006(2)$ & $0.025(2)$ & $-0.001(2)$ \\
$\mathrm{Nb} 2$ & $0.002(1)$ & $0.008(1)$ & $-0.007(1)$ & $0.006(2)$ & $0.005(2)$ & $-0.006(2)$ \\
$\mathrm{Nb3}$ & $0.012(2)$ & $0.053(1)$ & $-0.007(1)$ & $0.001(2)$ & $0.004(2)$ & $-0.001(2)$ \\
$\mathrm{Se} 1$ & $0.002(2)$ & $0.001(2)$ & $-0.004(2)$ & $0.013(3)$ & $0.003(2)$ & $-0.008(3)$ \\
$\mathrm{Se} 2$ & $0.001(2)$ & $-0.001(2)$ & $-0.004(2)$ & $-0.006(3)$ & $0.005(3)$ & $0.013(3)$ \\
$\mathrm{Se3}$ & $0.004(2)$ & $-0.002(2)$ & $-0.004(2)$ & $-0.010(3)$ & $0.012(3)$ & $0.012(3)$ \\
$\mathrm{Se} 4$ & $-0.001(2)$ & $-0.001(2)$ & $-0.002(2)$ & $0.004(3)$ & $0.001(3)$ & $0.001(3)$ \\
$\mathrm{Se} 5$ & $0.011(2)$ & $0.017(2)$ & $-0.006(2)$ & $0.004(3)$ & $0.000(2)$ & $-0.005(3)$ \\
$\mathrm{Se6}$ & $0.006(2)$ & $0.000(2)$ & $-0.007(2)$ & $0.005(3)$ & $0.012(3)$ & $0.001(3)$ \\
$\mathrm{Se} 7$ & $-0.007(2)$ & $0.021(2)$ & $0.031(2)$ & $0.001(3)$ & $0.001(3)$ & $-0.005(3)$ \\
$\mathrm{Se} 8$ & $0.021(2)$ & $-0.007(2)$ & $-0.027(2)$ & $0.001(3)$ & $0.003(3)$ & $0.001(3)$ \\
$\mathrm{Se} 9$ & $-0.002(2)$ & $0.014(2)$ & $0.021(2)$ & $-0.001(3)$ & $0.001(3)$ & $-0.002(3)$ \\
\hline
\end{tabular}


indicates a translation component of 0.5 along the appropriate fourth or fifth superspace coordinate.

The positions of the atoms are written as the sum of a lattice symmetric basic structure postion, $\mathbf{L}+\mathbf{x}^{0}$, with $\mathbf{L}$ a lattice vector, and a modulation function $\mathbf{u}\left(\bar{x}_{4}, \bar{x}_{5}\right)$. The latter can be written as a Fourier series in its arguments $\bar{x}_{4}=t_{1}+q_{1} \cdot x^{0}$ and $\bar{x}_{5}=t_{2}+q_{2}^{\prime} \cdot x^{0}$ :

$$
\mathrm{u}_{\alpha}\left(\bar{x}_{4}, \bar{x}_{5}\right)=\sum_{n} \sum_{m} A_{n, m}^{\alpha} \sin \left(2 \pi n \bar{x}_{4}+2 \pi m \bar{x}_{5}\right)+B_{n, m}^{\alpha} \cos \left(2 \pi n \bar{x}_{4}+2 \pi m \bar{x}_{5}\right)
$$

for $\alpha=1,2,3$. The summations run from zero to infinity, excluding the term $(n, m)=(0,0)$. As only first-order satellites for each modulation have been measured, only the Fourier terms $(1,0)$ and $(0,1)$ could be determined.

In the basic structure, all atoms are in a mirror plane at $y=0.25$ or at $y=0.75$. This gives rise to symmetry restrictions on the modulation functions. For the first harmonic, it is easy to derive that $B_{10}^{x}, A_{10}^{y}, B_{10}^{Z}$ are zero, as well as that $A_{01}^{x}, B_{01}^{y}, A_{01}^{z}$ are zero.

Refinement of the basic structure against the main reflections of crystal 1 resulted in a good fit with $R_{F}=0.073$, and position parameters only slightly different from the $100 \mathrm{~K}$ structure in reference 3 . The Refinements were done against $\left(\Sigma w\left|F_{\mathrm{O}}-\mathrm{F}_{\mathrm{c}}\right|^{2}\right)$, with weights given by $\mathrm{w}=1 /\left(\sigma_{\mathrm{F}}^{2}+\left(0.02\left|\mathrm{~F}_{\mathrm{O}}\right|\right)^{2}\right)$. Isotropic extinction correction was applied also. All calculations were performed with the refinement program JANA [9]. The temperature tensors did not change on introduction of the modulation. Several of them were not positive definite, as also was found at $100 \mathrm{~K}$ [3], which then is not related to the modulation.

Introduction of the 36 independent, first-order harmonic modulation parameters for each of the two CDW's, resulted in a good fit of the $\mathbf{q}_{1}$-type CDW and a reasonable fit of th $\mathbf{q}_{2}$-type CDW (Table 1). Coordinates, temperature factors, and modulation parameters are given in Tables 2 and 3. The isotropic extinction parameter refined to $\mathrm{G}=0.074(5)$.

The structure model as obtained from the refinement on the intensity data for crystal 1 was tested on the data set of crystal 2. As only a few reflections in a special part of reciprocal space $(\mathrm{k}=0)$ were measured for crystal 2 , only the scale factor and the extinction parameter were refined. Convergence was obtained after a few cycles, with R-factors given in Table 1 , and an isotropic extinction parameter $G=0.033(5)$. The good agreement between calculated and observed structure factors for the second crystal does give strong support for the structure model as determined on the data from crystal 1.

\section{Discussion}

The largest displacements were found on $\mathrm{Nb}$ (II) for the $\mathrm{q}_{1} \mathrm{CDW}$, and on $\mathrm{Nb}(\mathrm{I})$ for the $\mathrm{q}_{2} \mathrm{CDW}$ [7]. This is in accordance with the theoretical prediction of Wilson [10], NMR experiments [11, 12], and band structure calculations [17]. The displacements of the selenium atoms were interpreted as due to elastic coupling to the niobium atoms [7]. This then also involves major displacements of selenium atoms of column II. Scanning Tunneling Microscopy (STM) experiments have suggested that the $q_{2}-C D W$ resides on both type I and type II columns [18]. As STM mainly 


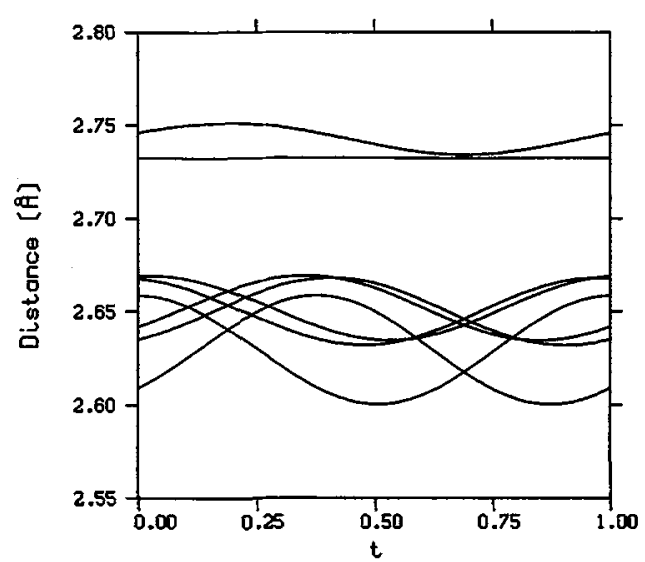

a.

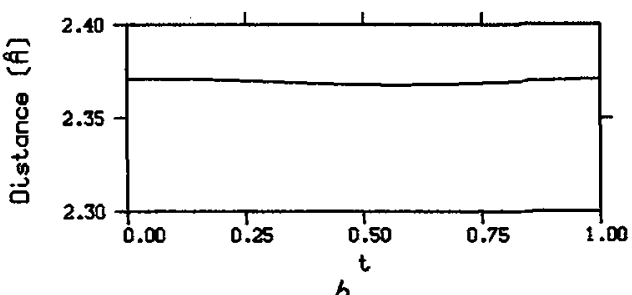

$b$

Figure 2. (a) Coordination of $\mathrm{Nb}(\mathrm{III})$ as a function of the phase $\bar{x}_{4}=t+q_{1} \cdot x^{\circ}$. The curves correspond to distances between $\mathrm{Nb}(\mathrm{III})$ and its eight neighboring $\mathrm{Se}$ atoms: Se7, Se8, and Se9, both at $y=-0.25$ and $y=0.75$, and Se8 and Se5. (b) The Se7 to Se9 distance as a function of $\bar{x}_{4}$.

Table 4. Formal valences as obtained with the Bond valence method. Parameters used are $r_{0}$ $=2.51 \AA$ and $\mathrm{b}=0.37 \AA[21]$.

\begin{tabular}{lccccccc}
\hline Atom & valence & \multicolumn{2}{c}{ Atom } & valence & \multicolumn{2}{c}{ Atom valence } & \multicolumn{2}{c}{ Atom valence } \\
\hline $\mathrm{Nb} 1$ & 5.28 & $\mathrm{Se} 1$ & 2.34 & $\mathrm{Se} 4$ & 1.46 & $\mathrm{Se} 7$ & 1.36 \\
$\mathrm{Nb} 2$ & 4.90 & $\mathrm{Se} 2$ & 1.33 & $\mathrm{Se} 5$ & 1.84 & $\mathrm{Se} 8$ & 2.30 \\
$\mathrm{Nb3}$ & 5.27 & $\mathrm{Se} 3$ & 1.45 & $\mathrm{Se} 6$ & 1.99 & $\mathrm{Se} 9$ & 1.37 \\
\hline
\end{tabular}

images the selenium atoms, the participation of $\mathrm{Se}$ (II) in the modulation provides a possible explanation for these observations [7]. Recent band-structure calculations indeed show the $q_{2}$ $\mathrm{CDW}$ to change the electronic states of both $\mathrm{Se}(\mathrm{I})$ and $\mathrm{Se}(\mathrm{II})$ at the energy and positions imaged by STM [19].

The correlated atomic displacements can be studied by considering the interatomic distances as a function of the additional superspace coordinates $\bar{x}_{4}$ and $\bar{x}_{5}$. Because the $\mathbf{q}_{1}$ and $\mathbf{q}_{2}$ CDW's are spatially resolved, both modulations can be analyzed independently. The $\mathbf{q}_{1}$ modulation affects the atoms around $\mathrm{Nb}(\mathrm{III})$. The maximum variation of the distances between $\mathrm{Nb}$ (III) and its eight neighboring selenium atoms is $0.06 \AA$ for Se8 (fig. 2a). This is much less than would occur for an out-of-phase displacements with the same amplitudes (variations up to $0.16 \AA$ ), thus showing the coherence between the $\mathrm{Nb}$ (III) and $\mathrm{Se}$ displacements. The remaining internal strain corresponds to the loss of elastic energy in the Peierls model. It is interesting to note that there is hardly any variation of the distance between $\mathrm{Nb}$ (III) and $\mathrm{Se} 5$ of column II: the latter atom completely follows the displacements of $\mathrm{Nb}(\mathrm{III})$. The absence of variation in the distance between $\mathrm{Se} 7$ and $\mathrm{Se} 9$ reflects the strong bonding in this $\mathrm{Se}_{2}^{2-}$ ion pair (fig. 2b). A similar picture is found for the $\mathrm{q}_{2}$ type modulation around $\mathrm{Nb}(\mathrm{I})$.

A simple electron count, based on the presence of $\mathrm{Se}_{2}^{2-}$ ion pairs and $\mathrm{Se}^{2-}$ ions, leads to 

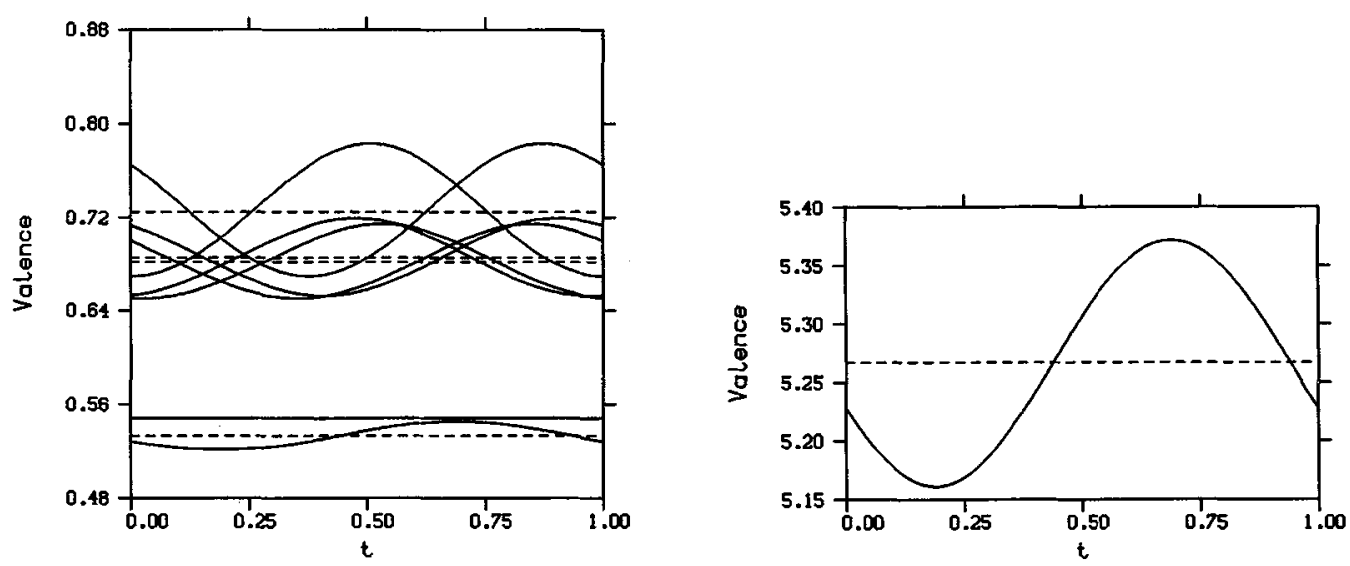

Figure 3. Valence of $\mathrm{Nb}$ (III) as a function of the phase $\bar{x}_{4}=t+q_{1} \cdot x^{0}$, calculated with the Bond-valence method [20,21]. (a) Individual Bond-valences for the same eight selenium atoms around $\mathrm{Nb}$ (III) as in fig. 2a. (b) The valence of $\mathrm{Nb}$ (III), as summed over the eight contributions in fig. 3a.

$\mathrm{Nb}$ (III) and $\mathrm{Nb}(\mathrm{I})$ being $4.2+$, while $\mathrm{Nb}$ (II) is $5.7+$. This analysis can be done more subtle using the Bond-valence method [20]. The valences of the Se atoms more or less follow the interpretation of $\mathrm{Se}^{2-}$ and $\mathrm{Se}_{2}^{2-}$ ions. Major difference is that $\mathrm{Se}$ of column II are calculated to be less negative than $\mathrm{Se}^{2-}$ ions. This results in $\mathrm{Nb}$ (II) being less positive than $\mathrm{Nb}(\mathrm{I})$ and $\mathrm{Nb}$ (III) (table 4), contrary to the model of Wilson [10] and the simple electron count. Bandstructure calculations exist which lead to either more positive or more negative $\mathrm{Nb}$ (II) [13-17]; valence-contrast diffraction suggested a more positive $\mathrm{Nb}$ (II) [22]. The Bond-valence calculation does show $\mathrm{Nb}$ (I) and $\mathrm{Nb}$ (III) to be nearly equivalent, but significantly different from $\mathrm{Nb}$ (II). Most noteworthy of the valence calculation is that it shows only a small difference in total bonding character of the three niobium atoms, in accordance with NMR results [23].

The valence of $\mathrm{Nb}(\mathrm{III})$ is calculated as 5.3, with a variation due to the $\mathrm{q}_{1} \mathrm{CDW}$ of 0.21 (fig.3b). The individual bond-valences show variations of about half this magnitude (fig.3a). With the coordination number 8 of each $\mathrm{Nb}$ it follows that these modulations compensate each other to a large extend. In particular this holds for each of the pairs of atoms Se7, Se8, and Se9, respectively below $(y=0.25)$ and above $(y=1.25) \mathrm{Nb}(\mathrm{III})$. Similarly, the variation in the selenium atom valences is much smaller than the sum of the variations of the bond-valences. These results are compatible with all atoms striving for a valence independent of the phase of the modulation wave. The $\mathrm{CDW}$ is then not reflected in a variation of the bonding character.

Partial support of this work by the National Science Foundation (CHE8711736) and support of the SUNY X3 beam line by the Division of Basic Energy Sciences of the U.S. Department of Energy (DEFG0286ER45231) are gratefully acknowledged. The National Synchrotron Light Source is supported by the U.S. Department of Energy, Division of Materials Sciences and Division of Chemical Sciences. 


\section{References}

[1] Meerschaut A. and Rouxel J., in: Crystal Chemistry and Properties of Materials with Quasi-One-Dimensional Structures, edited by J. Rouxel, Reidel, Dordrecht (1986).

[2] Monceau P., in: Electronic Properties of Inorganic Quasi-One-Dimensional compounds, Part II: Experimental, edited by P. Monceau, Reidel, Dordrecht (1985).

[3] Hodeau J.L., Marezio M., Roucau C., Ayroles R., Meerschaut A., Rouxel J. and Monceau P., J. Phys. C11 (1978) 4117.

[4] Tsutsumi K., Takagaki T., Yamamoto M., Shiozaki Y., Ido M., Sambongi T., Yamaya K. and Abe Y., Phys. Rev. Lett. 39 (1977) 1675.

[5] Fleming R.M., Moncton D.E. and McWhan D.B., Phys. Rev. B18 (1978) 5560.

[6] Fleming R.M., Moncton D.E., Axe J.D. and Brown G.S., Phys. Rev. B30 (1984) 1877.

[7] van Smaalen S., de Boer J.L., Meetsma A., Graafsma H., Sheu H.-S., Darovskikh A., Coppens P., and Levy F., Phys. Rev. B45 (1992) 3103.

[8] Levy F. and Berger H., J. Cryst. Growth 61 (1983) 61.

[9] Petricek V., Coppens P. and Becker P.J., Acta Cryst. A41 (1985) 478.

[10] Wilson J.A., Phys. Rev. B19 (1979) 6456.

[11] Devreux F., J. Phys. 43 (1982) 1489.

[12] Ross, Jr. J.H., Wang A. and Slichter C.P., Phys. Rev. Lett. 56 (1986) 663.

[13] Bullet D.W., J. Phys. C12 (1979) 277.

[14] Hoffmann R., Schaik S., Scott J.C., Whangbo M.-H. and Foshee M.J., J. Solid State Chem. 34 (1980) 263.

[15] Whangbo M.-H. and Gressier P., Inorg. Chem. 23 (1984) 1305.

[16] Shima N. and Kamimura H. in: Theoretical aspects of Band structures and electronic properties of pseudo-one-dimensional solids, edited by $\mathrm{H}$. Kamimura, Reidel, Dordrecht (1985).

[17] Canadell E., Rachidi I.E.-I., Pouget J.P., Gressier P., Meerschaut A., Rouxel J., Jung D., Evain M. and Whangbo M.-H., Inorg. Chem. 29 (1990) 1401.

[18] Dai Z., Slough C.G. and Coleman R.V., Phys. Rev. Lett. 66 (1991) 1318.

[19] Ren J. and Whangbo M.-H., Phys. Rev. B46 (1992) 4917.

[20] Brown I.D. and Altermatt D., Acta Cryst. B41 (1985) 244.

[21] Brese N.E. and O'Keeffe M., Acta Cryst. B47 (1991) 192.

[22] Gao Y., Pressprich M.R. and Coppens P., Acta Cryst. A49 (1993) 216.

[23] Shi J. and Ross Jr. J.H., Phys. Rev. B45 (1992) 8942. 